\title{
The distribution of the effects of genes affecting quantitative traits in livestock
}

\author{
Ben HAYES $^{\mathrm{a}, *}$, Mike E. GodDARD ${ }^{\mathrm{a}, \mathrm{b}}$ \\ ${ }^{a}$ Institute of Land and Food Resources, University of Melbourne, Parkville, \\ Victoria, 3052, Australia \\ ${ }^{\mathrm{b}}$ Department of Natural Resources and Environment, \\ Victorian Institute of Animal Science, \\ Attwood, Victoria, 3049, Australia
}

(Received 24 January 2000; accepted 2 January 2001)

\begin{abstract}
Meta-analysis of information from quantitative trait loci (QTL) mapping experiments was used to derive distributions of the effects of genes affecting quantitative traits. The two limitations of such information, that QTL effects as reported include experimental error, and that mapping experiments can only detect QTL above a certain size, were accounted for. Data from pig and dairy mapping experiments were used. Gamma distributions of QTL effects were fitted with maximum likelihood. The derived distributions were moderately leptokurtic, consistent with many genes of small effect and few of large effect. Seventeen percent and 35\% of the leading QTL explained $90 \%$ of the genetic variance for the dairy and pig distributions respectively. The number of segregating genes affecting a quantitative trait in dairy populations was predicted assuming genes affecting a quantitative trait were neutral with respect to fitness. Between 50 and 100 genes were predicted, depending on the effective population size assumed. As data for the analysis included no QTL of small effect, the ability to estimate the number of QTL of small effect must inevitably be weak. It may be that there are more QTL of small effect than predicted by our gamma distributions. Nevertheless, the distributions have important implications for QTL mapping experiments and Marker Assisted Selection (MAS). Powerful mapping experiments, able to detect QTL of $0.1 \sigma_{p}$, will be required to detect enough QTL to explain $90 \%$ the genetic variance for a quantitative trait.
\end{abstract}

distribution of gene effects / quantitative trait loci / genetic variance / marker assisted selection

\section{INTRODUCTION}

Traits of economic and ecological importance in livestock species are frequently quantitative. Both genetic and environmental variations contribute to the variation observed in quantitative traits in livestock populations. The

\footnotetext{
* Correspondence and reprints

E-mail: Ben.Hayes@nre.vic.gov.au
} 
genetic component of variation has been widely modelled assuming a large number of genes of small effect, termed the infinitesimal model. The infinitesimal model is attractive as it facilitates simple and elegant statistical descriptions of inheritance, such as predictable changes in genetic variance as a result of selection [5]. The discovery of a small number of genes of very large effect, such as the effect of the Hal gene on meat quality in pigs [17], led to a mixed model of inheritance of quantitative traits with many genes of small effect and rare genes of very large effect.

Recently, quantitative trait loci (QTL) of moderate effect have been found to be segregating even in selected populations $[2,9]$. We define a QTL as any gene having an effect of any measurable size on the quantitative trait. Detection of these QTL indicates the basic assumption of the infinitesimal model is flawed. Neither do the findings agree with the mixed model, which generally only accommodates single genes of very large effect. Then for a deeper understanding of the genetics of quantitative traits, information regarding the distribution of effects of QTL affecting quantitative traits is needed.

One source of information is from QTL mapping experiments. The aim of these experiments is to detect genes which contribute to quantitative trait variation, and determine their position on the chromosome. The livestock species with the most reported mapping information at present are pigs and dairy cattle. Results of four QTL mapping experiments with markers bracketing a large proportion of the porcine genome have been reported $[2,3,15,22$, 23]. Results of three QTL mapping experiments in dairy cattle with markers bracketing a large proportion of the bovine genome have been reported $[4,9$, 29]. At present, mapping experiments are not powerful enough to detect all the QTL that cause variation in quantitative traits, and QTL effects are only reported above a size determined by the experimental significance level. A second major limitation of using reported QTL effects to derive distributions of the effects of genes on quantitative traits is that effects reported are observed with experimental error.

In this paper we aim to derive distributions of QTL effects in pigs and dairy cattle using meta-analysis of published estimates of QTL effects. A QTL effect is defined as the effect of substituting the decreasing allele for the increasing allele. Dominance effects of the QTL are not considered for simplicity. The two major limitations of published estimates, that effects are observed with error, and only effects above a certain size for each experiment are reported, were accounted for. QTL effects were assumed to follow a gamma distribution. Gamma distributions are extremely flexible, and with only two parameters can describe any shape from equal gene effects to highly leptokurtic distributions [12]. As the total number of QTL detected in livestock species to date is limited, data from QTL experiments were accumulated across traits. The distributions of QTL effects derived are therefore for an "average" quantitative 
trait. Consequences of the distributions for QTL mapping experiments and Marker Assisted Selection (MAS) are explored.

\section{METHODS}

\subsection{Criteria for inclusion of data}

The literature was searched for results of QTL mapping experiments with markers covering a large proportion of the autosomal genome in pigs and dairy cattle. Data were the published estimates of QTL effects, and the standard errors of the effects. Within an experiment, data were included if the authors reported the QTL effect as significant, at the most stringent significance level used in that experiment. If no standard errors were presented but $P$ values were available, approximate standard errors were calculated from $P$ values using the $t$ distribution. If LOD scores were presented, these were converted to $P$ values using a $\chi_{1}^{2}$ distribution, and then to standard errors using the $t$ distribution. We assumed significant QTL reported in different experiments were different QTL, even if QTL mapped to approximately the same region. We made this assumption because at present mapping experiments are not precise enough to determine if QTL reported in different experiments map to exactly the same position on the genome.

\subsection{Pig data set}

Pig data were from crossbreeding experiments between divergent breeds. Three experiments generated $F_{2}$ progeny $[2,3,15]$, and one experiment generated backcross progeny $[22,23]$. The authors analysed their data assuming these breeds were fixed for alternate alleles at the QTL. The data extracted for this analysis were the additive effect of the QTL (half the difference between the two homozygotes) for significant QTL. Traits for which QTL were reported included growth, carcase and meat quality. The study of Andersson et al. [2] reported QTL effects as significant using a chromosome-wide significance level, whereas the other studies used a genome-wide significance level. Across all pig experiments, 32 significant additive QTL were reported.

\subsection{Dairy data set}

The three dairy experiments used a granddaughter design for QTL detection, with effects reported within grandsire families [28]. Data were gene substitution effects, the difference between the average effects of the two QTL alleles from the grandsire [8]. Gene substitution effects may include both additive and dominance effects. However in the within family designs used, additive and dominance effects could not be separated. When QTL effects 
were reported in daughter yield deviations (DYD), the effects were doubled to give the phenotypic effect. Traits for which QTL were reported were protein and fat percentage ( $\mathrm{P} \%, \mathrm{~F} \%)$, and protein, fat and milk yields (PY, FY, MY). Across all dairy experiments, 50 significant QTL effects were reported.

Effects for the \% traits reported by Georges et al. [9] were an order of 10 larger than effects for $\%$ traits reported in other studies, perhaps because they were actually in units of $\mathrm{g} \cdot \mathrm{L}^{-1}$. The phenotypic standard deviation for the $\%$ traits in Georges et al. [9] (derived from standard deviation of daughter yield deviations) was an order 10 larger than phenotypic standard deviations reported in other experiments. As a result, after QTL effects were scaled by phenotypic standard deviations, effects for \% traits in Georges et al. [9] were comparable with those elsewhere.

The QTL effect for MY reported by Zhang et al. [29] was an order of two larger than effects reported elsewhere. The phenotypic standard deviation for MY in Zhang et al. [29] was also large. After scaling, the QTL MY effect reported by Zhang et al. [29] was similar in magnitude to those reported elsewhere. The study of Zhang et al. [29] was unusual in that if QTL effects were detected in a number of families, only the largest and the mean (across families) QTL effects were reported. Only the largest QTL effects were reported with standard errors. As the standard errors of the QTL effects are required in our methods for deriving the distribution of QTL effects (see below), only these largest QTL effects were used. Some data are included in both the studies of Zhang et al. [29] and Georges et al. [9], but the published results were sufficiently different that data from both studies were included in the analysis.

The study of Ashwell et al. [4] used a substantially lower threshold for significant effects than other experiments.

In order to accumulate effects across traits within pig and dairy experiments, each effect and standard error were divided by the phenotypic standard deviation of the trait. If estimates of additive genetic variance, $V_{\mathrm{A}}$, and the environmental variance, $V_{\mathrm{E}}$, were reported with variance due to fixed effects removed, the phenotypic standard deviation used was $\sqrt{V_{\mathrm{A}}+V_{\mathrm{E}}}$. If $V_{\mathrm{A}}$ and $V_{\mathrm{E}}$ were not reported, the standard deviation of raw phenotypic records was used. For some of the dairy experiments, there was no information on phenotypic standard deviations for the traits, so literature estimates were used [18].

\subsection{Maximum likelihood estimation of distribution of QTL effects}

It was assumed that the true underlying QTL effects follow a gamma distribution, with scaling parameter $\alpha$ and shape parameter $\beta, g(x)=$ $\alpha^{\beta} x^{\beta-1} \mathrm{e}^{-\alpha x} / \Gamma(\beta)$. The first and second moments of the gamma distribution are $E(a)=\beta / \alpha$ and $E\left(a^{2}\right)=\beta(\beta+1) /\left(\alpha^{2}\right)$. The kurtosis $\left(\gamma^{2}\right)$ of the distribution was calculated as $\gamma^{2}=(\beta+2)(\beta+3) /(\beta(\beta+1))$. For example, 
$\beta \rightarrow \infty$ is the limiting case for all effects being equal, in which case $\gamma^{2}=1$. Conversely, as $\beta \rightarrow 0, \gamma^{2} \rightarrow \infty$, and the distribution becomes increasingly leptokurtic [12], and skewed to the right with many effects close to zero.

The estimated effect of the QTL, reported in the literature, was assumed to follow a normal distribution. The mean of the normal distribution was the true QTL effect, and the standard deviation was the estimation error for the QTL. Let $n\left(\widehat{x_{i, j}} \mid x\right)$ be the ordinate of the normal distribution for $\widehat{x_{i, j}}$, the $j$ th observed effect from the $i$ th experiment, given the true QTL effect is $x$. Then

$$
n\left(\widehat{x_{i, j}} \mid x\right)=\frac{1}{\sqrt{2 \pi} \sigma_{i}} \mathrm{e}^{-\left(\frac{\left(\widehat{x_{i, j}}-x\right)^{2}}{2 \sigma_{i}^{2}}\right)}
$$

where the average standard error for experiment $i$ is $\sigma_{i}$. All variables are expressed in units of phenotypic standard deviations.

We define the QTL effect as the effect of substituting the decreasing allele with the increasing allele, so all substitution effects were positive. It is possible that the observed QTL effect and true QTL effect have opposite signs (i.e. a negative QTL effect is observed when the true QTL effect is positive). The experimenter has no way of knowing that this has occurred. Therefore the value of the normal distribution when observed effects were given the opposite sign to the true QTL effect, $n\left(-\widehat{x_{i, j}} \mid x\right)$ was included to complete the distribution. A density function for $\widehat{x_{i, j}}$ can be written as

$$
f\left(\widehat{x_{i, j}}\right)=\int_{0}^{\infty} n\left(\widehat{x_{i, j}} \mid x\right) g(x) \mathrm{d} x+\int_{0}^{\infty} n\left(-\widehat{x_{i, j}} \mid x\right) g(x) \mathrm{d} x .
$$

We also took into account the fact that QTL are only observed above a truncation point determined by the significance threshold for each experiment. The truncation point, $c_{i}$, was taken as the value of the smallest of the significant observed effects in each experiment. Then the probability that $\widehat{x_{i, j}}$ is observed when the true QTL effect is gamma distributed with parameters $\alpha$ and $\beta$ is,

$$
P\left(\widehat{x_{i, j}} \mid \alpha, \beta\right)=\frac{f\left(\widehat{x_{i, j}}\right)}{\int_{c_{i}}^{\infty} f(\hat{x}) \mathrm{d} \hat{x}} .
$$

The log likelihood for all $\widehat{x_{i, j}}$ in $t$ experiments, with $m_{i}$ significant QTL effects in experiment $i$, is $\sum_{i=1}^{t} \sum_{j=1}^{m_{i}} \ln P\left(\widehat{x_{i, j}} \mid \alpha, \beta\right)$.

Numerical integration was used to integrate the distributions where required. A grid search was used to find the maximum likelihood (ML) estimates of $\alpha$ and $\beta$ given the data. 
Support limits for parameters $\alpha, \beta, E(a)$ and $E\left(a^{2}\right)$ were obtained by linear interpolation from differences in log likelihood from the maximum over the profile. The fitted parameter value corresponding to a change in log likelihood of 2 was taken as the support limit, which is asymptotically equivalent to a $95 \%$ confidence limit [12]. Kurtosis was calculated from the maximum likelihood estimate of $\beta$.

\subsection{Number of heterozygous QTL per sire}

In this section we attempt to calculate the total number of heterozygous QTL per sire. Only heterozygous QTL can be detected in mapping experiments. We assume that the number of observed QTL above the significance threshold is equal to the number of true QTL above the significance threshold. We realise this is unlikely to be the case, as some of the observed QTL could be false positives. The error introduced by this assumption is reduced somewhat by using only QTL reported as significant at stringent significance thresholds.

The number of QTL per trait per sire observed in an experiment was $n_{i}$ for experiment $i$. The QTL above size $c_{i}$ are a proportion of the total QTL. This proportion can be calculated as $p_{i}=\int_{c_{i}}^{\infty} f(\hat{x}) \mathrm{d} \hat{x}$. Therefore the total number of heterozygous QTL per trait per sire or $F_{1}$ boar is

$$
N_{i}=\frac{n_{i}}{p_{i}}
$$

The number of heterozygous QTL per trait per sire or $\mathrm{F}_{1}$ boar was calculated from each of the pig and dairy experiments. The average number of heterozygous QTL per sire for each of the species was calculated, as

$$
\bar{N}=\frac{\sum_{i=1}^{t} n_{i}}{\sum_{i=1}^{t} p_{i}} .
$$

The value of $n_{i}$ was adjusted before calculating $\bar{N}$ for (i) the proportion of the genome bracketed by markers, and (ii) in dairy data only, for the probability that a grandsire is heterozygous for a marker bracket surrounding a QTL. Sections of the genome were assumed to be bracketed by markers if there was less than $50 \mathrm{cM}$ between adjacent markers. Assumptions regarding the number of QTL per marker bracket depend on the assumptions made by authors of the papers used in the meta-analysis, but generally a maximum of one QTL per bracket per experiment was assumed. Pitfalls of this assumption are considered in the discussion. 
For dairy, we only predicted the number of heterozygous QTL for Georges et al. [9] and Zhang et al. [29]. Ashwell et al. [4] only used 16 markers in their study, and the proportion of the genome bracketed by these markers could not be reliably predicted. Georges et al. [9] estimated their marker map covered $66 \%$ of the genome. If $n_{i}=0.5$ with $66 \%$ of the genome bracketed by markers, $n_{i}=0.76$ would be expected if $100 \%$ of the genome were bracketed. Zhang et al. [29] used markers which bracketed almost the entire autosomal genome so no adjustment to $n_{i}$ was necessary (their estimate).

In the mapping experiments used to provide data for our meta-analysis, the heterozygosity of markers was not $100 \%$. Therefore some sires may have been homozygous for marker brackets containing heterozygous QTL. If a sire is homozygous for a marker, it is not possible to detect a heterozygous QTL linked to that marker. As an approximation, we have assumed that the proportion of sires that are detected as heterozygous at the QTL is proportional to the average heterozygosity of the markers, and adjusted $n_{i}$ accordingly. The average heterozygosity of markers used in the experiments of Georges et al. [9] and Zhang et al. [29] were $45.8 \%$ and $46.1 \%$ respectively. The numbers of heterozygous QTL/sire/trait detected in these experiments were 0.14 and 0.36 for Georges et al. [9] and Zhang et al. [29] respectively. Given our assumption, if the markers in each of these experiments were $100 \%$ heterozygous, 0.31 and $0.78 \mathrm{QTL} /$ sire/trait would have been detected.

As the pig experiments used an across family analysis, QTL were reported per trait rather than per trait per sire. Therefore no adjustment to $n_{i}$ for the average heterozygosity of markers in sires was used.

\subsection{Within sire segregation variance}

The within sire segregation variance explained by the distribution of QTL effects is derived as follows. For each experiment, the variance caused by the segregation of $N_{i}$ genes within the gametes from one sire is $N_{i} \frac{1}{4} E\left(a^{2}\right)$.

For dairy experiments, the amount of within sire segregation variance which the distribution of QTL effects explains can be compared to the within sire segregation variance. The within sire variance can be calculated from typical heritability estimates, as $1 / 4 h^{2}$. In pig experiments, $N_{i} \frac{1}{4} E\left(a^{2}\right)$ is equivalent to the $F_{1}$ segregation variance.

\subsection{Total number of QTL segregating in the population}

In outbred populations, one individual will only be heterozygous for fraction $(2 K)$ of the total number of genes segregating. Given the number of QTL found heterozygous in each sire $\left(N_{i}\right)$, the total number of segregating loci $(M)$ will be given by $M=N / 2 K$. An estimate of $M$ is therefore $M=\bar{N} / 2 K$. We can calculate $2 K$ by making some assumptions about the distribution of gene 
frequencies. We assume the distribution of gene frequencies is for a population previously without selection for the quantitative trait, and with all genes neutral with respect to fitness. We recognise that these assumptions are not correct, particularly if the population has undergone some artificial selection, in which case QTL with large effects are likely to be at extreme frequencies. In this case we are likely to underestimate $2 K$, the proportion of heterozygous QTL. However, the assumptions allow us to provide a rough estimate of the number of genes explaining the variance in quantitative traits. Given our assumptions, distribution of gene frequencies will reflect the generation of new alleles by mutation and their loss by drift. The gene frequency probability density from this assumption is $f(p)=K /(p(1-p))$, where $K$ is a constant and $p$ is the frequency of one allele [14]. This calculates the gene frequency distribution accurately if the product of the mutation rate per locus and effective population size is small. This is likely to be the case in most livestock populations. The relevant part of the gene frequency distribution is from $\pi \leqslant p \leqslant 1-\pi$, where $\pi=1 /\left(2 N_{e}\right)$. The value of $\pi$ is the lowest possible gene frequency in a population of effective size $N_{e}$. We have used effective population size as an approximation. In fact all parents in the population are targets for mutation. In modern livestock populations however, mutations may only be exploited if they occur in elite breeding animals (e.g. AI sires in the dairy industry), as it is these animals which provide genetic material for the improvement of the population. The size of the elite population is likely to be nearer the effective population size than the census population size.

The constant, $K$, is chosen so that

$$
\int_{\pi}^{1-\pi} K /(p(1-p)) \mathrm{d} p=1 .
$$

Integrating this function and solving for $K$ gives the result,

$$
K=\frac{1}{2 \ln \left(\frac{1-\pi}{\pi}\right)} .
$$

The mean heterozygosity of QTL is

$$
\int_{\pi}^{1-\pi} 2 p(1-p) K /(p(1-p)) \mathrm{d} p
$$

which can be approximated as $2 K$, or

$$
\frac{1}{\ln \left(\frac{1-\pi}{\pi}\right)} \text {. }
$$




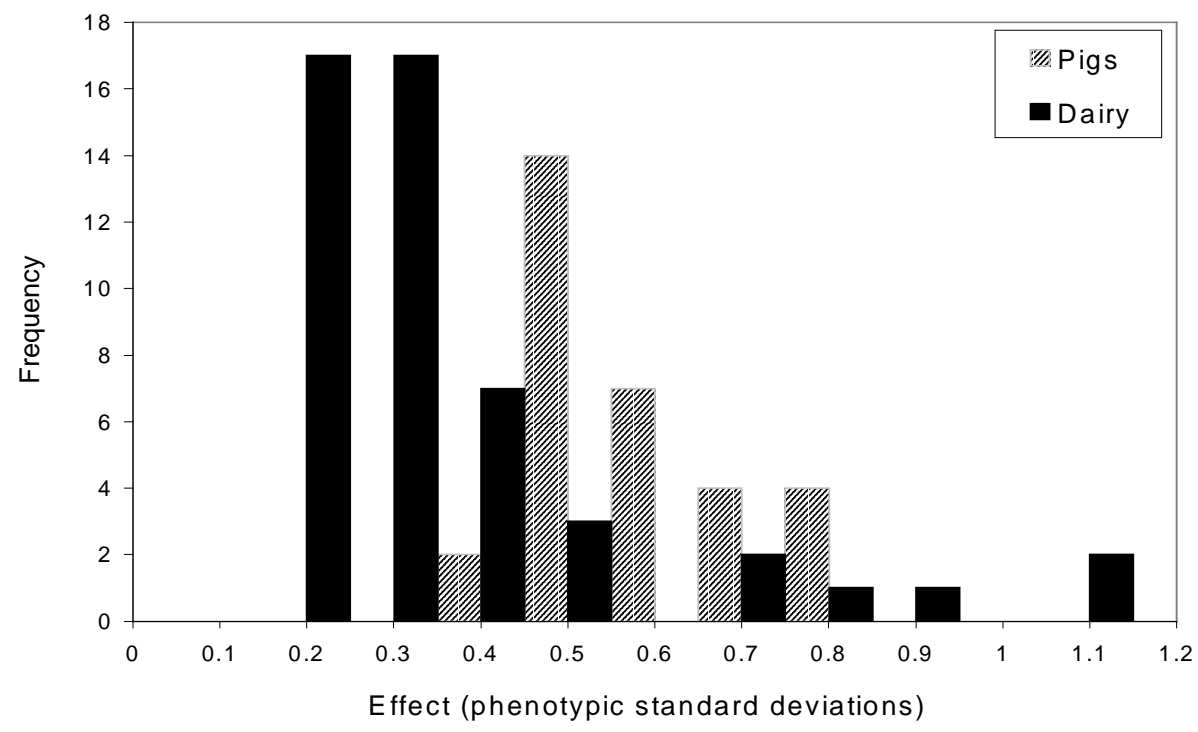

Figure 1. Distribution of additive (QTL) effects from pig experiments, scaled by the standard deviation of the relevant trait, and distribution of gene substitution (QTL) effects from dairy experiments scaled by the standard deviation of the relevant trait.

This is the mean heterozygosity among the loci that are segregating and depends only on the effective population size. The total number of QTL segregating in the population was calculated with effective population sizes of 50,500 and 5000 ( $\pi=0.01, \pi=0.001$ and $\pi=0.0001$ respectively). The number of QTL was only calculated from the dairy data. The pig resource populations used in the mapping experiments were not suitable for calculating the total number of QTL segregating in the population, as the number of segregating QTL calculated from wide crosses would be unlikely to be representative of commercial pig populations.

\section{RESULTS}

\subsection{Distribution of QTL effects}

The frequency distribution of apparent QTL effects from pig experiments and dairy distributions accumulated over traits and scaled by the phenotypic standard deviation of each trait is shown in Figure 1.

The pig distribution indicates a greater number of QTL of moderate size (e.g. between $0.3 \sigma_{p}$ and $0.5 \sigma_{p}$ ) have been detected than large QTL $\left(e . g .>0.5 \sigma_{p}\right)$. The raw average QTL effect was $0.42 \sigma_{p} \pm 0.02 \sigma_{p}$.

For the dairy frequency distribution, a greater number of QTL of moderate to small size have been detected than large QTL. The number of relatively 
Table I. Maximum likelihood estimates for gamma distributions of QTL effects.

\begin{tabular}{cll}
\hline Parameter (Support limits) & \multicolumn{1}{c}{ Pig } & \multicolumn{1}{c}{ Dairy } \\
\hline$\alpha$ & $11.2(7.8-15.6)$ & $5.4(3.6-7.8)$ \\
$\beta$ & $1.48(0.30-3.02)$ & $0.42(0.18-0.78)$ \\
$E(a)$ & $0.1321(0.0736-0.2262)$ & $0.0778(0.0409-0.1357)$ \\
$E\left(a^{2}\right)$ & $0.0293(0.0105-0.0721)$ & $0.0205(00787-0.0507)$ \\
$\gamma^{2}$ & 4.25 & 13.88 \\
\hline
\end{tabular}

small QTL (e.g. $\left.<0.3 \sigma_{p}\right)$ was larger in the dairy data set than the pig data set. Dairy experiments generally have more power to detect small QTL than the pig experiments. The raw average QTL effect for dairy data was lower than for the pig data, $0.32 \sigma_{p} \pm 0.03 \sigma_{p}$.

Support limits were large for the ML estimates of parameters of scale $(\alpha)$ and shape $(\beta)$ for the gamma distribution for pig and dairy effects, Table I. The large support limits reflect the small size of pig and dairy data sets.

We used a likelihood ratio test to determine if the pig and dairy distributions were significantly different. The gamma distribution was fitted to a data set containing both pig and dairy data. ML parameters for this pooled data set were scale $(\alpha)=7.1$ and shape $(\beta)=0.59$. The likelihood ratio was calculated as $-2^{*}$ the natural logarithm of the ratio of the sum of the maximum likelihood's of the pig and dairy data analysed separately to the maximum likelihood of the pooled data set. The likelihood ratio was compared to a chi-squared statistic with one degree of freedom at the 0.05 significance level. The distributions were not significantly different. However, the small size of the data sets means that the distributions would have to be very different before the likelihood ratio test was significant.

The first moment of the distribution is the mean QTL effect. The mean QTL effect from the pig and dairy gamma distributions were much smaller than the raw mean of the QTL data.

Both distributions were moderately leptokurtic, and implied many QTL of small effect, and few of large effect, Figure 2.

Figure 2B suggests a greater density of QTL above $1 \sigma_{p}$ for dairy than for pigs. This is agreement with the frequency distributions for pig and dairy QTL effects scaled by phenotypic standard deviations (Fig. 1).

The variance contributed by the QTL of effect greater than a specified truncation point was determined. To do this we assumed that large QTL and small QTL will have similar frequency distributions. Figure 3A plots $\int_{c}^{\infty} x^{2} g(x) \mathrm{d} x$ against $c$, where $c$ is a specified truncation point. As QTL effects are observed with error, the apparent variance explained by observed QTL may be different to actual genetic variance explained by true QTL. The apparent 
$\mathbf{A}$

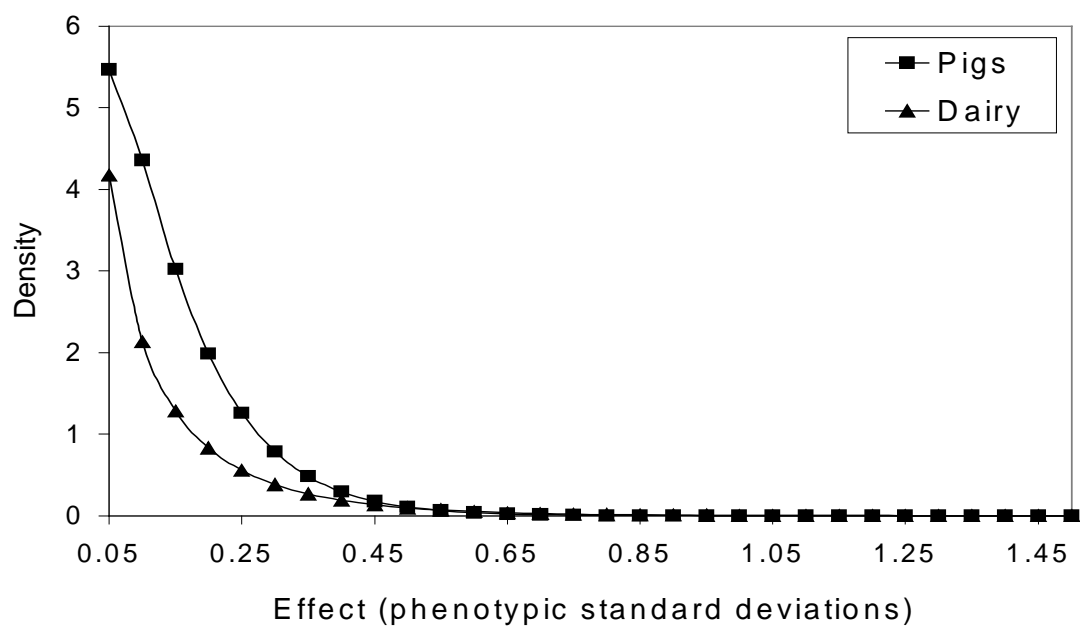

B

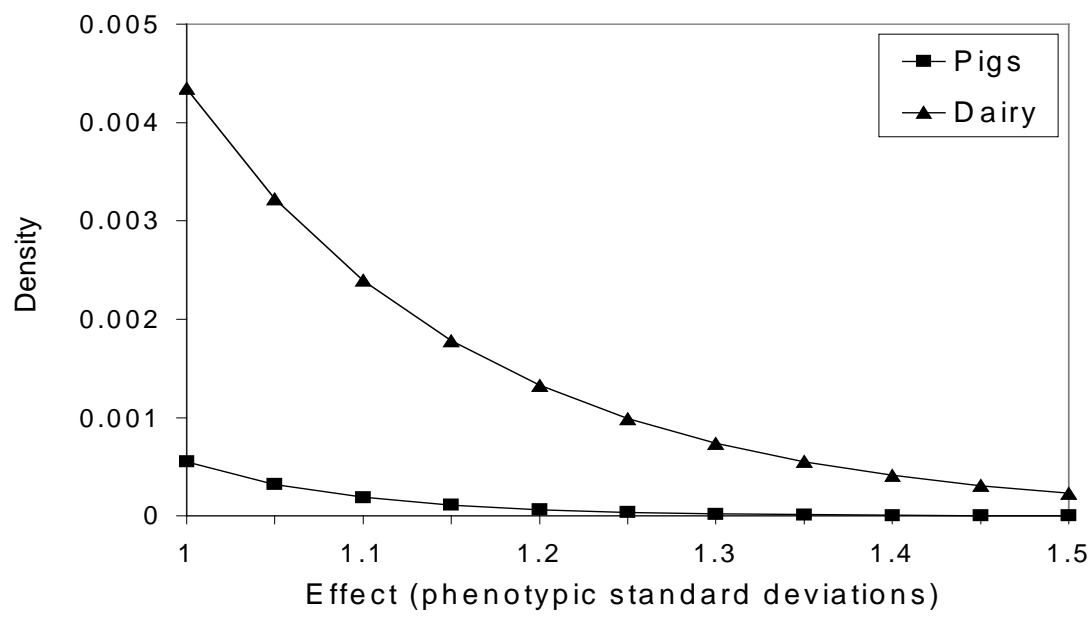

Figure 2. Distributions of QTL effect from pig and dairy experiments (A), and magnification of distribution from effect $=1$ to $1.5 \sigma_{p}(\mathrm{~B})$.

variance explained by observed QTL above a truncation point can be determined by plotting $\int_{c}^{\infty} \hat{x}^{2} f(\hat{x}) \mathrm{d} \hat{x}$ against $c$, Figure $3 \mathrm{~B}$.

Figure $3 \mathrm{~A}$ suggests QTL $>0.1 \sigma_{p}$ contribute approximately $90 \%$ of the variance for both species. The figure also suggests for dairy, the segregation of large QTL $\left(>1 \sigma_{p}\right.$ ) explains about $5 \%$ of the total variance. For pigs, the proportion of the variance explained by the segregation of large QTL is very small. This is because our distributions predict the occurrence of large QTL is very rare for pigs. 
A

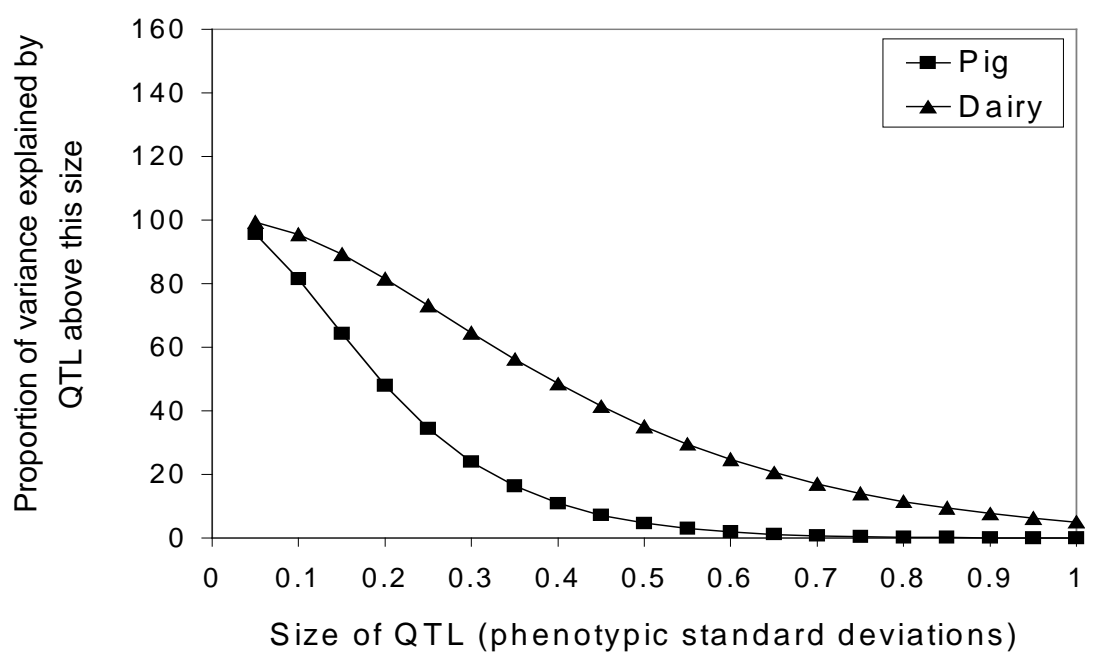

B

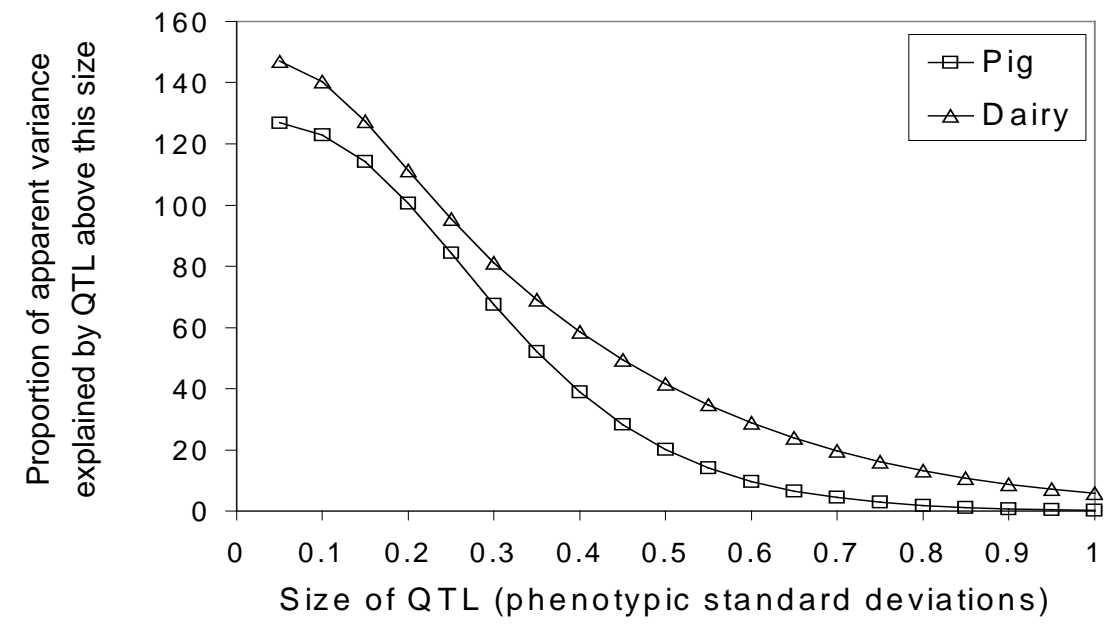

Figure 3. Variance contributed by the QTL above a specified size, (A) estimated proportion of variance when size of QTL is size of the true QTL effect, and (B) variance which would be observed when size of QTL effect is size of the observed effect.

Figure 3B indicates the apparent variance explained by observed QTL will be greater than the true variance explained. The apparent variance explained is greater than the true variance explained because QTL effects are overestimated in mapping experiments [9]. For example the true variance explained by QTL greater than $0.35 \sigma_{p}$ for dairy is $55 \%$, while the apparent variance explained by QTL greater than $0.35 \sigma_{p}$ is $70 \%$. 


\subsection{Number of heterozygous QTL per sire}

The estimated number of heterozygous QTL per sire per trait was approximately 10 for both pig and dairy distributions, Table II.

For dairy, the results are consistent between studies. For pigs, the number of segregating QTL per trait are approximately similar for Roher and Keele [22,23], Andersson et al. [2] and Knott et al. [15]. The numbers of segregating QTL predicted from Andersson-Eklund et al. [3] is much lower. Andersson-Eklund et al. [3] included many more meat quality traits in their experiment than the other experiments. Meat quality traits typically have a low heritability [24]. This could indicate a problem with pooling results over different traits.

The average number of QTL heterozygous in $F_{1}$ boars was similar to the average number of QTL heterozygous in dairy sires. At first sight this is surprising since the $F_{1}$ boar is a wide cross and the dairy sires are largely pure Holstein. However the pig experiments were analysed assuming that alternate alleles were fixed in the parent pig breeds so that all $\mathrm{F}_{1} \mathrm{~s}$ were heterozygous and identical. This is probably an unrealistic assumption, with the consequence that the mapping experiments may fail to detect QTL which are heterozygous in some $F_{1}$ boars but not in others.

\subsection{Within sire segregation variance}

The within sire segregation variance estimated for dairy cattle was 0.055 . The average number of heterozygous QTL/sire/trait from Table II was used to make this prediction. Thus the distribution represents a generic trait with heritability of 0.22 . For comparison, if heritabilities from literature are averaged over the five dairy production traits (using $h^{2}$ for percentage traits as 0.5 and $h^{2}$ for yield traits as 0.2$) 1 / 4 h^{2}$ is 0.080 [18]. Hence the predicted value of within sire segregation variance underestimates the average of estimates from the literature by about $30 \%$.

Using the number of genes in Table II for each pig experiment, the $F_{1}$ segregation variances were calculated as 0.056 [22,23], 0.124 [2], 0.108 [15] and 0.035 [3]. The differences between studies can be explained by the different traits which were included in the mapping experiments. Both Andersson et al. [2] and Knott et al. [15] examined growth and carcass traits, which have moderate to high heritability. Andersson-Eklund et al. [3] examined a large number of carcass and meat quality traits, with moderate to low heritability. The $F_{1}$ segregation variance for these traits could be expected to be lower than the $F_{1}$ segregation variance for the traits examined by Andersson et al. [2] and Knott et al. [15]. Roher and Keele [22,23] examined fat deposition and muscling and wholesale product yield traits, which have moderate to high heritability. 


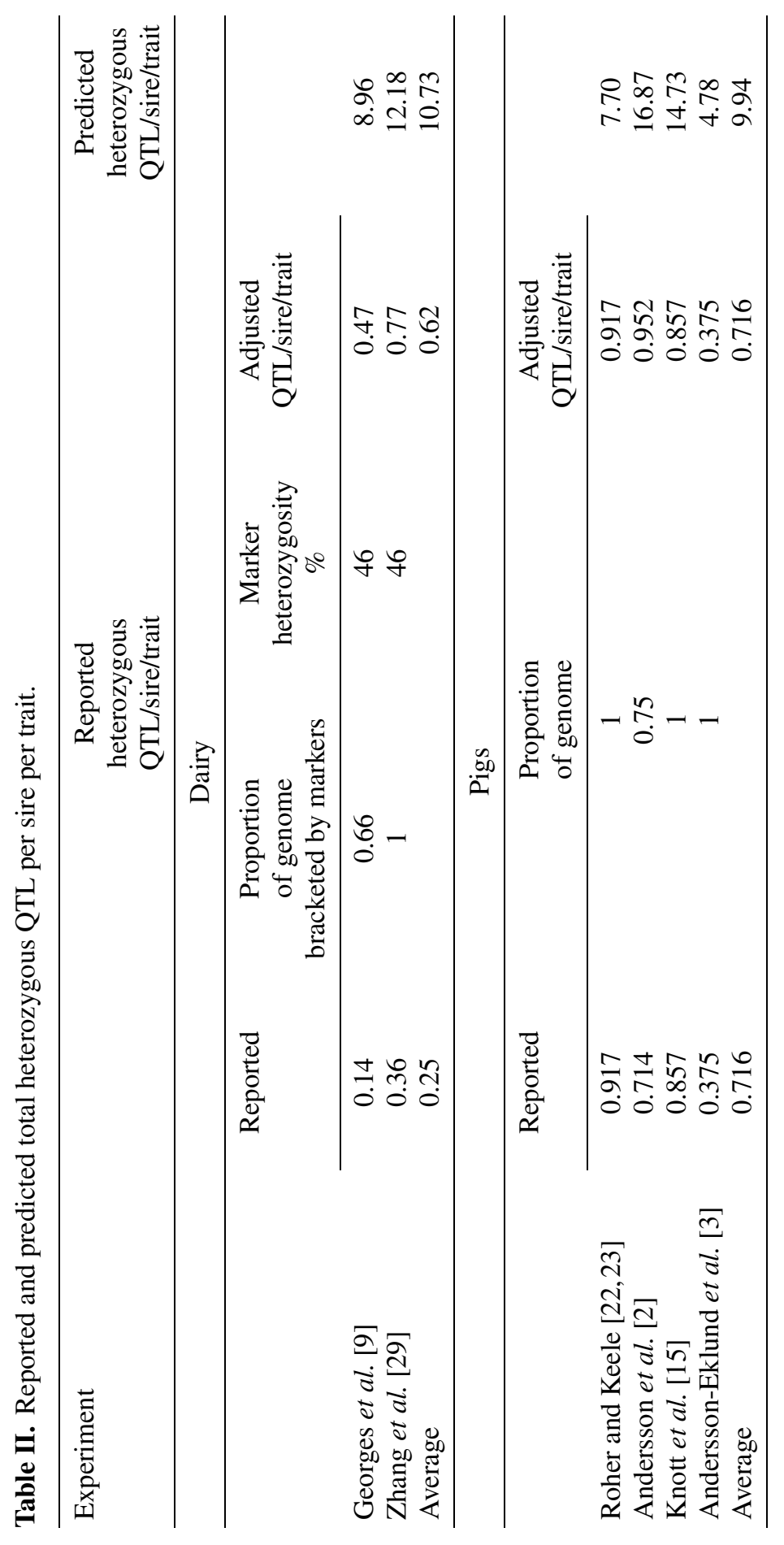




\subsection{Total number of segregating QTL in the population}

The number of segregating QTL was predicted to be 49, 74 and 99 for effective population sizes of 50,500 and 5000 respectively. It is difficult to say which estimate is the most likely. The world's dairy population is increasingly dominated by the Holstein breed. In addition, widespread use of Artificial Insemination (A.I.) has reduced the number of bulls used to breed dairy sires worldwide. As a result, the effective population size of the world's dairy herd is relatively low [10], but this is a recent phenomenon and the effective population size was probably larger in the past.

\subsection{Expected true QTL effects given observed effects}

The distribution of effects of QTL on quantitative traits can be immediately applied to correct bias in the QTL effects reported in literature. Bias in reported effects arises because QTL effects are only reported above a size determined by the experimental significance level, and effects are observed with experimental error, and so effects that reach the significance threshold and are reported are likely to be overestimated $[9,29]$.

The expected true QTL effect, given an observed QTL effect, is,

$$
E(x \mid \hat{x})=\int_{0}^{\infty} x f(x \mid \hat{x}) \mathrm{d} x
$$

which can be calculated as,

$$
\frac{\int_{0}^{\infty} x n(\hat{x} \mid x) g(x) \mathrm{d} x}{\int_{0}^{\infty} n(\hat{x} \mid x) g(x) \mathrm{d} x}
$$

However, this does not take account of the large number of chromosome segments with no heterozygous QTL. In a typical mapping experiment, 100 chromosome segments may be tested for the presence of QTL. We have estimated that approximately 10 heterozygous QTL are segregating per sire. Hence 90\% of the chromosome segments do not contain QTL, and therefore have a true effect of zero. An effect can still be observed for these chromosome segments due to experimental error. The expected true QTL effect, given an observed QTL effect, is then,

$$
E(x \mid \hat{x})=\frac{0.1 \int_{0}^{\infty} x n(\hat{x} \mid x) g(x) \mathrm{d} x}{0.1 \int_{0}^{\infty} n(\hat{x} \mid x) g(x) \mathrm{d} x+0.9 n(\hat{x} \mid 0)} .
$$




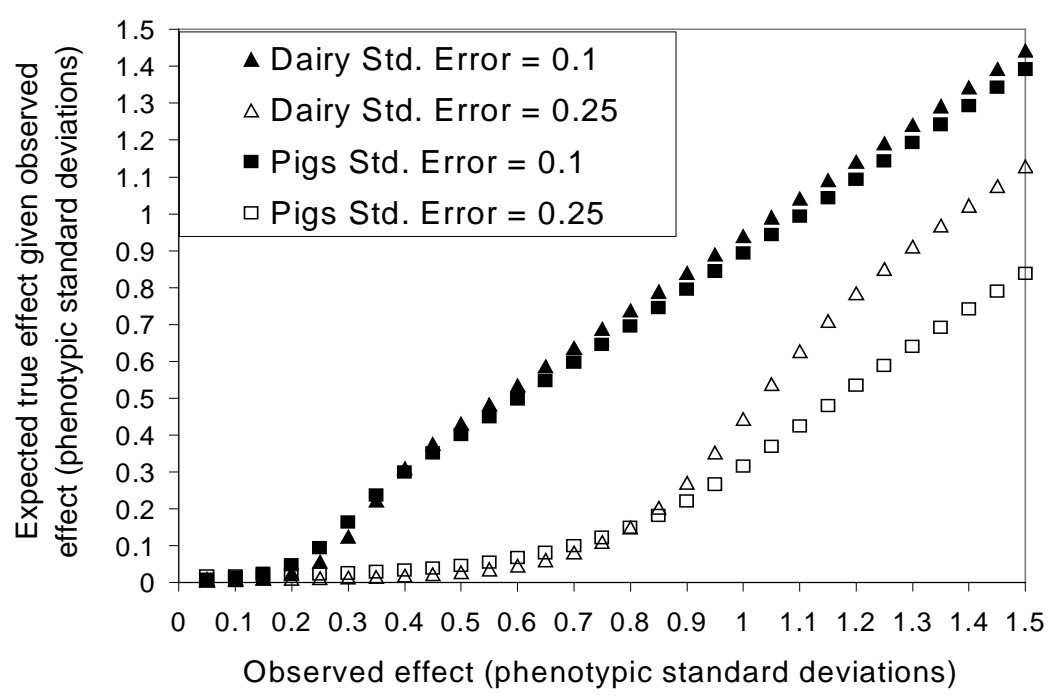

Figure 4. Expected true QTL effect given an observed QTL effect with standard errors $0.1 \sigma_{p}$ and $0.25 \sigma_{p}$.

Where $n(\hat{x} \mid 0)$ is the ordinate of the normal distribution at $\hat{x}$ with mean zero and standard deviation the standard error of the experiment.

The value of $E(x \mid \hat{x})$ can then be plotted against the observed QTL effects for the pig and dairy distributions, given specified standard errors (Fig. 4).

Even with an experimental standard error of $0.1 \sigma_{p}$, the figure indicates observed QTL of small to moderate effect (e.g. $0.1 \sigma_{p}$ to $0.3 \sigma_{p}$ ) will be serious overestimates of the true QTL effect. Observed QTLs of this size have a high probability of being chromosome segments which in fact contain no QTL, with the observed effect purely due to experimental error.

Figure 4 indicates observed QTL of moderate effect (e.g. $0.4 \sigma_{p}$ ) will also be serious overestimates, by $22 \%$ and $25 \%$ for dairy and pigs respectively, when the standard error is $0.1 \sigma_{p}$. Large observed QTL (e.g. $\left.1 \sigma_{p}\right)$ are overestimates of the true QTL effect by approximately $5-10 \%$, if the standard error is $0.1 \sigma_{p}$. However if the standard error is large $\left(0.25 \sigma_{p}\right)$, as was the case with most observed QTL of effect $>1 \sigma_{p}$, the overestimation of the size of the QTL is more serious, at $55 \%$ for dairy and $70 \%$ for pigs.

\section{DISCUSSION}

The limitations of the data on which this meta-analysis is based must be acknowledged. The limited number of significant QTL reported in the literature (32 for pigs and 50 for dairy) meant support limits for parameters of distributions of QTL effects were large. Data quality was compromised by failure of 
some studies to report phenotypic variances and other necessary information for the resource population. In these cases the phenotypic standard deviation was taken from other sources, and were therefore only approximations. Despite this, internal consistency of the data was high. The magnitude of QTL effects scaled by phenotypic standard deviations generally in good agreement for traits across studies.

Since the data for the analysis included no QTL of small effect, the ability to estimate the number of QTL of small effect must inevitably be weak. The within sire segregation variance explained by the distribution estimated for dairy traits appears to under estimate the true within-sire segregation variance. It may be that there are more QTL of small effect than predicted by our gamma distributions. In addition, the numbers of QTL segregating are predicted from our distribution with low accuracy, given the large support limits for parameters of the distributions. An example illustrates how the number of genes changes from the support limits to the ML estimates for the distribution. Assume 0.62 QTL/sire/trait are detected in an experiment with a truncation point of $0.35 \sigma_{p}$, with an average standard error of $0.11 \sigma_{p}$ (similar to the average values for the dairy experiments). Then using the ML estimates for $\alpha$ and $\beta$ from the dairy distribution, 11.8 heterozygous QTL per sire are predicted. Using the distributions at either extreme of the joint (for $\alpha$ and $\beta$ ) 95\% confidence interval from the ML surface gives an interval of 21 heterozygous QTL per sire to 6 heterozygous QTL per sire.

For pigs, the $F_{1}$ segregation variance estimated from the gamma distribution of gene effects appears to be less than half the heritability that might be expected in an $F_{2}$. This may be for the reasons discussed in the previous paragraph, but could also be because only QTL with alternative alleles fixed in the parent breeds are being detected.

The difference in the shape of the distributions estimated from the pig and dairy data could be due to sampling errors. However differences could also be due to the designs of the experiments. The pig experiments sought QTL which had different alleles fixed in each parent breed. If the differences between breeds were partly due to selection, then one would expect fixation of alleles to occur more often for alleles of medium to large effect than small effects.

The QTL effects reported in the literature are actually for chromosome segments, rather than individual genes. Our distributions could therefore be more correctly termed distributions of effects of chromosome segments in livestock species, rather than distributions of the effects of genes. Our predictions of the number of segregating QTL are actually predictions of the number of segregating chromosome segments with an effect on the quantitative trait in a population. If there are two or more segregating genes within a chromosome segment bracketed by markers, our methods will underestimate the number of QTL segregating. 
While distributions for QTL effects in livestock species have not previously been reported, it is useful to compare our results to those from laboratory species and from theoretical models of variation in quantitative traits. There is increasing evidence in laboratory species for leptokurtic distributions of gene effects. Shrimpton and Robertson [25] isolated chromosome segments, or polygenic factors, which controlled bristle score in Drosophila melanogaster. Although their experiment could not ascertain factors less than 0.6 phenotypic standard deviations in size, they concluded their observations supported the hypothesis that quantitative characters are controlled by a few major effects supported by a host of smaller effects of diminishing influence on the character. This agrees with the shape of our distributions. The shape of our distributions is also similar to, but somewhat less leptokurtic than, those observed for mutation effects in laboratory species $[12,19,20]$.

There are no estimates in the literature of the number of heterozygous loci affecting a quantitative trait segregating in pig and dairy populations to compare to our results. We can however, compare our values to literature estimates for the number of genes controlling quantitative characters in other species. Lande [16] used the Wright index [6] to estimate the minimum number of loci controlling fruit weight in tomato, percent oil in maize kernels, fish eye diameter, and date of anthesis in Goldenrod. The average number of effective loci over all traits and species was approximately 10 , with a range of 5 to 22. However, Wright's estimate assumes all allelic effects are equal. This is unlikely given the shape of our distributions for genes affecting quantitative traits. We can adjust Wrights Index to accommodate unequal allelic effects that follow the distributions predicted in this study. After making this adjustment, the average number of loci over all traits and species in Lande [16] would be 30. This value is less than, but close to, the number of QTL we predict are segregating in dairy populations (if the effective population size is small). It is important to note that we have not predicted the total number of genes in the genome with an effect on a quantitative trait, but only the number of genes which are segregating in the population. The total number of genes which affect a trait will be many times larger.

Our distributions of effects of QTL on quantitative traits can be compared to distributions which have emerged from theoretical models of variation in quantitative traits. Wahlroos et al. [26] simulated populations with finite numbers of loci over many generations of mutation - natural selection drift followed by a few generations of artificial selection. The variance in these populations after artificial selection was largely generated by a small proportion of QTL of intermediate effect $\left(0.2 \sigma_{p}<a<0.6 \sigma_{p}\right)$. This agrees with our results, e.g. Figure 3.

The distribution of effects of QTL on a quantitative trait has utility for planning QTL mapping experiments. The aim of mapping experiments is to detect 
QTL which contribute to genetic variance. Most QTL detection experiments performed to date have had a significance threshold of approximately $0.35 \sigma_{p}$ or greater (e.g. $[9,15,29])$. Even if QTL of this size could be observed without error in a mapping experiment, Figure 3A indicates all the QTL detected would only explain $58 \%$ of the variance for dairy, and $20 \%$ of the variance for pigs. To detect enough QTL to explain 90\% of the variance, experiments would need to detect QTL as small as $0.1 \sigma_{p}$. Weller et al. [28] estimate to detect QTL with effects of this size with a power of 0.73 would require a grandsire design with 20 grandsires, 200 sons per grandsire and 100 granddaughters per son. This is a considerably larger experiment than the mapping experiments which have been performed to date $[4,9,29]$.

Recently, a number of researchers have used finite locus models to estimate additive variance and breeding values [7,11,13,21]. The models assume a prior distribution of gene effects from which effects at individual loci are sampled. Uniform distributions [7], Normal distributions [7,11,21], exponential distributions [21] and gamma distributions [13] have been assumed. In all cases, the parameters of the distributions were chosen arbitrarily. The distribution of QTL effects proposed here would be a natural candidate for the distribution of gene effects used in such models.

Quantitative trait loci mapping experiments are at an early stage, and few significant results have been reported to date. Results from many QTL mapping experiments will be published in the next few years. We recommend results from these experiments be included in data sets for the different species, and distributions of effects of QTL on quantitative traits be periodically re-estimated as more information becomes available.

A current trend in the human genetic literature is to use meta-analysis of linkage data across a number of studies (e.g. Allison and Heo [1]). A single such meta-analysis has been carried out in pigs to estimate the effect and location of a QTL affecting backfat on chromosome four [27]. Such approaches increase the power to discriminate between false positive and true QTL. Estimates of QTL effects from meta-analysis would be valuable data for estimating the distribution of QTL effects, as estimation errors of the QTL effects should be lower relative to data from single studies.

\section{ACKNOWLEDGEMENTS}

The author's are grateful for funding from a grant from the Pig Research and Development Corporation (PRDC), grant number US43, "MARKER ASSISTED SELECTION FOR PROFITABLE PIGS", to complete this research. Two anonymous reviewers are thanked for valuable comments on an earlier version of the manuscript. 


\section{REFERENCES}

[1] Allison D.B., Heo M., Meta-analysis of linkage data under worst case conditions: a demonstration using the human OB region, Genetics 148 (1998) 859-865.

[2] Andersson L., Haley C.S., Ellegren H., Knott S.A., Johansson M. Andersson K., Andersson-Eklund L., Edfors-Lilja I., Fredholm M., Hansson I., Hakansson J., Lundstron K., Genetic mapping of quantitative trait loci for growth and fatness in pigs, Science 263 (1994) 1771-1774.

[3] Andersson-Eklund L., Marklund L., Lundstrom K., Haley C.S., Andersson K., Hansson I., Moller M., Andersson L., Mapping quantitative loci for carcass and meat quality traits in a wild boar $\times$ large white intercross, J. Anim. Sci. 76 (1998) 694-700.

[4] Ashwell M.S., Rexroad C.E. Jr., Miller R.H., VanRaden P.M., Da Y., Detection of quantitative trait loci affecting milk production and health traits in an elite US Holstein population using microsatellite markers, Anim. Genet. 28 (1997) 216-222.

[5] Bulmer M.G., The effect of selection on genetic variability, Am. Nat. 105 (1971) 201-211.

[6] Castle W.E., An improved method of estimating the number of genetic factors concerned in cases of blended inheritance, Science 54 (1921) 223.

[7] Du F.X., Hoeschele I., Estimation of additive, dominance and epistatic variance components using finite locus models implemented with a single site Gibbs and a descent graph sampler, Genet. Res. 76 (2000) 187-198.

[8] Falconer D.S., Introduction to quantitative genetics, 3rd edn., Longman, UK, 1989.

[9] Georges M., Nielson D., Mackinnon M., Mishra A., Okimoto R., Pasquino A.T., Sargeant L.S., Sorenson A., Steele M.R., Zhao X., Womack J.E., Hoeschele I., Mapping quantitative trait loci controlling milk production in dairy cattle by exploiting progeny testing, Genetics 139 (1995) 907-920.

[10] Goddard M.E., Optimal effective population size for the global population of black and white dairy cattle, J. Dairy Sci. 75 (1992) 2902-2911.

[11] Goddard M.E., Gene based models for genetic evaluation - an alternative to BLUP?, in: Proceedings of the 6th World Congress on Genetics Applied to Livestock Production, 1998, Vol. 26, Armidale, University of New England, pp. 33-36.

[12] Keightley P.D., The distribution of mutation effects on viability in Drosophila melanogaster, Genetics 138 (1994) 1315-1322.

[13] Kerr R.J., Henshall J.M., Tier B., Use of a finite locus model to estimate genetic parameters is unselected populations, in: Proceedings of the 13th Conf. Assoc. Advanc. Anim. Br. Genet., 1999, Mandurah, pp. 404-407.

[14] Kimura M., Crow J.F., The number of alleles that can be maintained in a finite population, Genetics 49 (1964) 725-738.

[15] Knott S.A., Marklund L., Haley C.S., Andersson K., Davies W., Ellegren H., Fredholm M., Hansson I., Hoyheim B., Lundstrom K., Moller M., Andersson L., Multiple marker mapping of quantitative trait loci in a cross between outbred wild boar and large white pigs, Genetics 149 (1998) 1069-1080. 
[16] Lande R., The minimum number of genes contributing to quantitative variation between and within populations, Genetics 99 (1981) 541-553.

[17] Lundstrom K., Karlsson A., Hakansson J., Johansson M., Andersson L., Andersson K., Production, carcass and meat quality traits of $\mathrm{F}_{2}$-crosses between European Wild Pigs and domestic pigs including halothane gene carriers, Anim. Sci. 61 (1995) 325-331.

[18] Misztal I., Lawlor T.J., Short T.H., Van Raden P.M., Multiple-trait estimation of variance components of yield and type traits using an animal model, J. Dairy Sci. 75 (1992) 544-551.

[19] Mukai T., Chigusa S.I, Mettler L.E., Crow J.F., Mutation rate and dominance genes affecting viability in Drosophila melanogaster, Genetics 72 (1972) 333355.

[20] Oshni O., Spontaneous and ethyl methanesulfante induced mutations controlling viability in Drosophila melanogaster. Ph. D. Thesis, University of Wisconsin, 1974.

[21] Pong-Wong R., Haley C.S., Woolliams J.A., Behaviour of the additive finite locus model, Genet. Sel. Evol. 31 (1999) 193-211.

[22] Roher G.A., Keele J.W., Identification of quantitative trait loci affecting carcass composition in swine. I. Fat deposition traits, J. Anim. Sci. 76 (1998) 2247-2254.

[23] Roher G.A., Keele J.W., Identification of quantitative trait loci affecting carcass composition in swine. II. Muscling and wholesale product yield traits, J. Anim. Sci. 76 (1998) 2255-2262.

[24] Rothschild M.F., Ruvinsky A., The genetics of the pig, CAB International, Wallingford, 1998.

[25] Shrimpton A.E., Robertson A., The isolation of polygenic factors controlling bristle score in Drosophila melanogaster. II. Distribution of third chromosome bristle effects within chromosome sections, Genetics 118 (1988) 445-459.

[26] Wahlroos H., Caballero A., Maki-Tanila A., Distribution of QTL in selected populations under a non-additive pleiotropic model, in: 49th Annual Meeting of the European Association for Animal Production, 23-27 August 1998, Warsaw, Poland.

[27] Walling G.A., Visscher P.M., Andersson L., Rothschild M.F., Wang L., Moser G., Groenen M.A.M., Bidanel J., Cepica S., Archibald A.L., Geldermann H., de Koning D.J., Milan D., Haley C.S., Combined analyses of data from quantitative trait loci mapping studies: chromosome 4 effects on porcine growth and fatness, Genetics 155 (2000) 1369-1378.

[28] Weller J.L., Kashi Y., Soller M., Power of granddaughter designs for determining linkage between marker loci and quantitative trait loci in dairy cattle, J. Dairy Sci. 73 (1990) 2525-2537.

[29] Zhang Q., Boichard D., Hoeschele I., Ernst C., Eggen A., Murkve B., PfisterGenskow M., Witte L.A., Grignola F.E., Uimari P., Thaller G., Bishop M.D., Mapping quantitative trait loci for milk production and health of dairy cattle in a large outbred pedigree, Genetics 149 (1998) 1959-1973. 